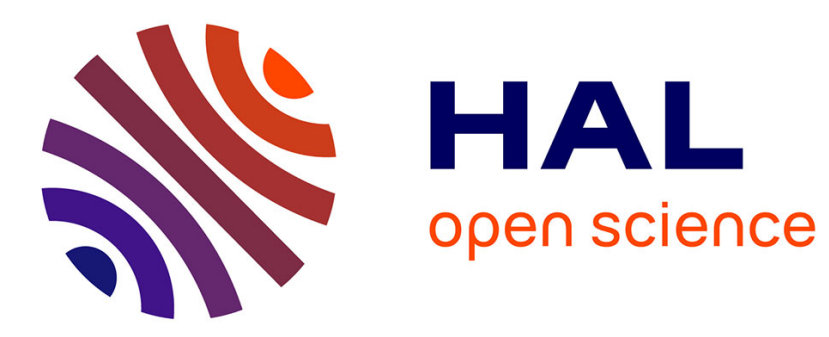

\title{
Motorcycle Speed Profile in Cornering Situation
}

Hamid Slimi, Hichem Arioui, Lydie Nouvelière, Saïd Mammar

\section{To cite this version:}

Hamid Slimi, Hichem Arioui, Lydie Nouvelière, Saïd Mammar. Motorcycle Speed Profile in Cornering Situation. American Control Conference (ACC 2009), Jun 2010, Baltimore, MD, United States. pp.1172 -1177. hal-00515560

\section{HAL Id: hal-00515560 \\ https://hal.science/hal-00515560}

Submitted on 7 Sep 2010

HAL is a multi-disciplinary open access archive for the deposit and dissemination of scientific research documents, whether they are published or not. The documents may come from teaching and research institutions in France or abroad, or from public or private research centers.
L'archive ouverte pluridisciplinaire HAL, est destinée au dépôt et à la diffusion de documents scientifiques de niveau recherche, publiés ou non, émanant des établissements d'enseignement et de recherche français ou étrangers, des laboratoires publics ou privés. 


\title{
Motorcycle Speed Profile in Cornering Situation
}

\author{
H. Slimi, H. Arioui, L. Nouveliere and S. Mammar
}

\begin{abstract}
In this paper, we present a new method for the computation of the maximal authorized motorcycle speed in curves. The three main factors which are the vehicle, the driver and the infrastructure are taken into account.

The vehicle dynamics are represented by a four degrees of freedom model which includes the vehicle's longitudinal slip and sideslip angle. The driver behavior model considers the ability in deceleration maneuvers according to the mobilized friction. The infrastructure characteristics introduce a precise handling of the road geometry and of the maximal available friction.
\end{abstract}

\section{INTRODUCTION}

$\mathbf{S}$ INCE 2002, the French government decided to make the fight against the road accidents one of the large programs of its actual period. In spite of encouraging results obtained since the installation of speed control systems, the motorcycle remains a particularly dangerous mode of transport: the number of death is still very high, and if one takes account of the number of traveled kilometers, the risk of death for a motorcycle rider is 21 times higher than that of other transportation modes.

Even if motorcycles are now experimenting several electronic equipments such as front-rear coupled braking, ABS and ESC systems on the top range models, accident still occur because of inadequacy between the dynamics, the driver inputs and infrastructure characteristics. In fact, the driver could generally surprised by sudden change in road curvature or banking angle his inputs could exhibit overreaction. It is thus of primary importance to develop preventive passive warning and active safety systems.

Under these facts, the aim of the national research programs, such as SUMOTORI and E-MOTIVE is to overcome partly these deficiencies. More precisely, their objective relates to the three following aspects:

- To acquire knowledge on the behavior of the two wheeled vehicles in accident situations.

- To prove the feasibility of an onboard system which will be able to detect critical situations to overcome accident if possible?

- To design a demonstrator of this onboard system in order to warn the rider and/or to activate passive safety systems.

H. Slimi, H. Arioui, L. Nouveliere and S. Mammar are with IBISC-CNRS Fre 3190 Laboratory of Evry Val d'Essonne University, 91020 Evry Cedex, France hamid.slimidibisc.univ-evry.fr
This paper is focussed on the development of a computational method of the motorcycle maximal speed authorized for driving an upcoming curve. The interaction of the three actors which are the motorcycle, the driver and the infrastructure are taken into account. The motorcycle dynamics are represented by a model which includes longitudinal load transfer. The driver behavior considers the ability in deceleration while the infrastructure characteristics introduce a precise handling of the road geometry (curvature, slope and road bank angle) and the maximal available friction.

The remainder of the paper is organized as follow: the problem statement is provided in section 2. Existing speed models in curves are reviewed in section 3 while the proposed speed profile model is detailed in section 4. Simulation results are provided is section 5. The conclusions in Section 6 wrap up the paper.

\section{Problem Statement}

The long-term objective of this study is to present a proposal to assist drivers of two-wheeled vehicles by warning them sufficiently in advance in order to anticipate dangerous situations [1]. Recall that since 20 years about 1600 people are killed each year in motorcycle accident [2]. The proposed assistance architecture is depicted in figure.

The warning system is interacting with the triplet $\mathrm{D}$ V-I (Driver-Vehicle-Infrastructure) via a human-machine interface. In the triplet D-V-I, the driver influences the dynamic's motorcycle by his actions (Torque, roll and lean angles), the road also affects the vehicle through tire/road contact, and dictates the desired trajectory for a driver.

This present work is dedicated for the generation of a maximal authorized speed profile, in cornering situation, which will be used as adynamic threshold in a warning system. In fact an alarm delivered when this limit is reached.

The warning system exploits the information provided by different physical sensors to have knowledge on the changes in the motorcycle dynamics and parameters. However, the state and the parameters are usually not directly measurable: sensors do not exist yet, or they are prohibitively expensive. In this case observers are used [8]. However for the sake of simplicity, in this paper the information will be considered as available. 


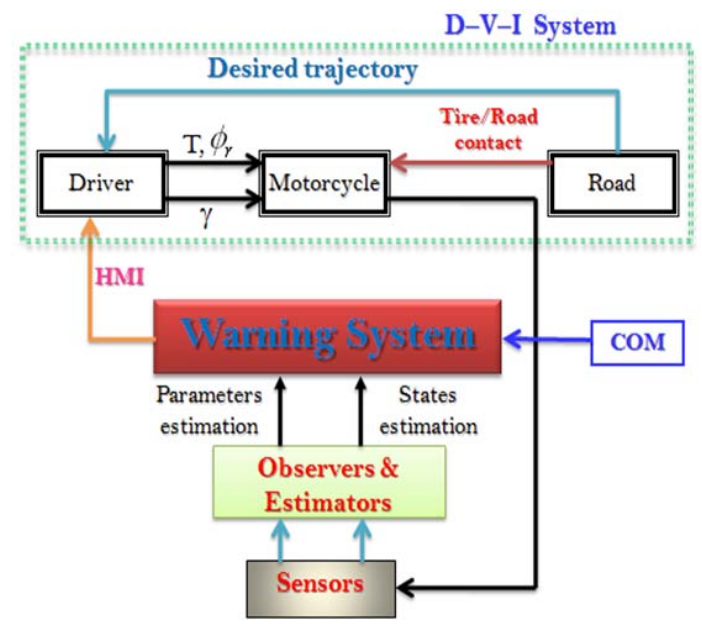

Fig. 1. Architecture of the warning system [1]

\section{Existing Speed Models in Curves}

The maximum safe-speed in a curve depends on the road geometry, the surface conditions, the driver's skills (or tolerance for discomfort) and the rollover stability of the vehicle. The geometric factors of a curve that are always fixed are the radius of curvature, the slope and the super-elevation or banking. The other road-dependent factor is the maximum lateral friction factor that can be generated by the road surface. The friction factor can vary from vehicle to vehicle; it varies also with the temperature and the wetness of the surface, the tires operating conditions and the vehicle speed.

Neglecting any other parameter than the road curvature $\rho$, the maximum speed at which a vehicle can be kept on the road while moving at a constant speed on a circular section is given by:

$$
v_{\text {max }}=\sqrt{\frac{g \cdot \mu_{\text {lat }}}{\rho}}
$$

Where, $g$ is the acceleration due to gravity and $\mu_{\text {lat }}$ is the maximum available side friction.

The friction coefficient $\mu_{\text {lat }}$ depends on the tire-road contact characteristics.

The determination of the safe-speed in a curve is generally based on the information on lateral acceleration.

This speed limit expresses in particular, the maximum road curvature according to acceptable maximum lateral acceleration which is between $0.2 g$ and $0.3 g$ according to safety and comfort margins, [3].

The National Highway Traffic Safety Administration (NHTSA), in [3], recommends for the design and development of Curve Warning Systems that the maximum safe speed at the apex of an approaching curve is determined by the following equation:

$$
v_{\text {max }}=\sqrt{\frac{g}{\rho}\left(\frac{\phi_{r}+\mu_{\text {lat }}}{1-\phi_{r} \mu_{\text {lat }}}\right)}
$$

In the NHTSA model includes the road super-elevation angle $\phi_{r}$ which compensates in part the lateral acceleration. The description of the road is thus more precise compared to the first one.

Furthermore, by taking into account the super-elevation $\phi_{r}$, thr slope $\phi_{p}$ and driver behavior one can give a more precise model. Indeed, in previous work [1], a new formula was presented (equation 3) for the calculation of the maximum authorized speed $v_{x}$ in a curve. This speed takes into account the road geometry and driver behavior:

$$
\left\{\begin{array}{l}
\phi_{p} \geq 0 \\
v_{x}^{2}=\frac{g}{\rho}\left\{\left(1-\frac{h}{l_{f}} \phi_{p}\right) \sqrt{1-\left(\frac{\phi_{p}}{\lambda_{\text {long }} \mu_{\max }}\right)^{2}} \cdot \lambda_{\text {lat }} \cdot \mu_{\text {max }}-\phi_{r}\right\} \\
\phi_{p}<0 \\
v_{x}^{2}=\frac{g}{\rho}\left\{\left(1-\frac{h}{l_{f}} \phi_{p}\right) \sqrt{1+\left(\frac{\phi_{p}}{\lambda_{\text {long }} \mu_{\max }}\right)^{2}} \cdot \lambda_{\text {lat }} \cdot \mu_{\max }-\phi_{r}\right\}
\end{array}\right.
$$

where $\phi_{p}$ is the road slope, $\phi_{r}$ the super-elevation, $\lambda_{\text {lat }} \cdot \mu_{\max }$ (resp. $\lambda_{\text {long }} \cdot \mu_{\max }$ ) represents the side friction (resp. longitudinal friction) mobilized by the driver, $h$ is the height of the vehicle centre of gravity and $l_{f}$ (resp. $l_{r}$ ) is the distance between the centre of gravity and the front axe (resp. rear axe) .

In this model, the vehicle dynamics are not entirely taken into account.

In the following an enhancement of this model is considered.

\section{New Approach For Speed Generation}

In [1], we give a new method for the computation of the maximum authorized roll angle of a motorcycle driving in a curve. This proposed approach takes into account the driver and the geometry of the road. However, the vehicle dynamics are not entirely taken into account. The dynamic model enhancements are:

- A two bodies motorcycle model which takes into account at the same time the forces at each of the tire/road contact points and the load transfer due to the longitudinal accelerations.

- Lateral and longitudinal mobilized frictions on each wheel are computed. The highest safe speed is defined considering the maximum friction. As we consider a four degrees of freedom model, its speed is computed by resolving the maximum of the two tires mobilized frictions.

- The driver deceleration profile. 


\section{A. Motorcycle Model}

The tire is an important part of the motorcycle which is difficult to model. The road-tire contact role is the transmission of the guidance forces. A simplified dynamic model of a motorcycle with two bodies that is sufficiently precise to approximate lateral dynamics in the curves is choses. Longitudinal dynamics (Wheel-slip) are also added to this model [4], [7], [9], [12] and [13].

The model has two joined rigid bodies and is inspired from those presented in [6] and [5].

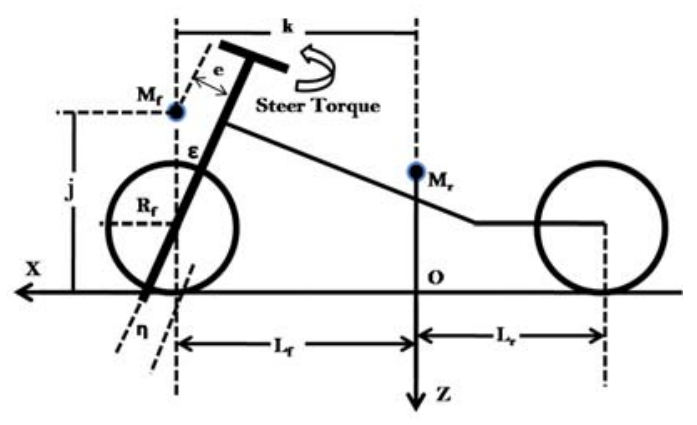

Fig. 2. Schematic diagram of the Motorcycle

The longitudinal dynamics are given by:

$$
\begin{aligned}
& M(\ddot{x}-\dot{y} \cdot \dot{\psi})-\left(M_{f} \cdot j+M_{r} \cdot h\right) \cdot \dot{\phi} \cdot \dot{\psi}^{2}-\left(M_{f} \cdot e \cdot \cos (\varepsilon)\right) \cdot \dot{\delta}^{2} \\
& -F_{\text {aerolong }}-M \cdot g \cdot \sin \left(\phi_{r}\right) \cdot \sin \left(\phi_{p}\right)=\sum F_{x i}
\end{aligned}
$$

Where $\sum F_{x i}$ represents the longitudinal forces acting on the tires and which is according to the longitudinal slip, $F_{\text {aerolong }}$ is the aerodynamic force in the longitudinal direction.

$$
\sum F_{x i}=F_{x f}+F_{x r}=\sum K_{p i} \cdot \lambda_{i}
$$

where $i=1,2, K_{p i}$ is the longitudinal stiffness coefficient of the front and rear tire, $\lambda_{i}$ is the longitudinal slip of the front and rear tire.

The lateral motion is described by four equations as follows:

$$
\begin{aligned}
& M(\ddot{y}+\dot{x} \cdot \dot{\psi})+\left(M_{f} \cdot j+M_{r} \cdot h\right) \cdot \ddot{\phi}+\left(M_{f} \cdot k\right) \cdot \ddot{\psi}+\left(M_{f} \cdot e\right) \ddot{\delta} \\
& -F_{\text {aerolat }}+M \cdot g \cdot \cos \phi_{p} \sin \phi_{r} \\
& =\sum F_{y i} \\
& \left(M_{f} \cdot j+M_{r} \cdot h\right) \ddot{y}+a_{1} \ddot{\phi}+a_{2} \ddot{\psi}+a_{3} \ddot{\delta}+a_{4} \cdot \dot{x} \cdot \dot{\psi}+a_{5} \cdot \dot{x} \cdot \dot{\delta} \\
& =\sum M_{x} \\
& \left(M_{f} \cdot k\right) \cdot(\ddot{y}+\dot{x} \cdot \dot{\psi})+a_{2} \ddot{\phi}+b_{1} \ddot{\psi}+b_{2} \ddot{\delta}-b_{3} \cdot \dot{x} \cdot \dot{\phi}-a_{5} \cdot \dot{x} \cdot \dot{\delta} \\
& =\sum M_{z} \\
& \left(M_{f} \cdot e\right) \ddot{y}+a_{3} \ddot{\phi}+c_{1} \ddot{\psi}+c_{2} \ddot{\delta}-a_{5} \cdot \dot{x} \cdot \dot{\phi}+c_{3} \cdot \dot{x} \cdot \dot{\psi} \\
& =\sum M_{s}
\end{aligned}
$$

with:

$$
\begin{aligned}
& \sum M_{x}=\left(M_{f} \cdot j+M_{r} \cdot h\right) \cdot g \cdot \sin \phi-\left(\eta \cdot Z_{f}-M_{f} \cdot e \cdot g\right) \cdot \sin \delta \\
& \sum M_{z}=l_{f} \cdot F_{f}-l_{r} \cdot F_{r}-\tau \cdot \cos \varepsilon \\
& \sum M_{s}=-\left(\eta \cdot Z_{f}-M_{f} \cdot e \cdot g\right) \cdot \sin \phi \\
& -\left(\eta \cdot Z_{f}-M_{f} \cdot e \cdot g\right) \cdot \sin \varepsilon \cdot \sin \delta+\tau
\end{aligned}
$$

where, $F_{\text {aerolat }}$ is the lateral aerodynamic force, $\Sigma F_{y i}$ is the sum lateral forces exerted on each tire. These forces can be considered as proportional to the tire slip angle and camber angle. One can express the lateral forces on the two tires as:

$$
\left\{\begin{array}{l}
F_{y f}=-C_{f 1} \cdot \alpha_{f}+C_{f 2} \cdot \phi_{f} \\
F_{y r}=-C_{r 1} \cdot \alpha_{r}+C_{r 2} \cdot \phi_{r}
\end{array}\right.
$$

$C_{f i}$ and $C_{r j}$ are the lateral stiffness coefficients of the tire respectively and the camber coefficients of the tire.

On the other hand, the slip angles of the front and rear wheel are given by:

$$
\left\{\begin{aligned}
\alpha_{f} & =\left(\frac{v_{y}+l_{f} \cdot \dot{\psi}-\eta \cdot \dot{\delta}}{v_{x}}\right)-\delta \cdot \cos (\varepsilon) \\
\alpha_{r} & =\left(\frac{v_{y}-l_{r} \cdot \dot{\psi}}{v_{x}}\right)
\end{aligned}\right.
$$

$l_{f}$ (resp. $l_{r}$ ) represents the distance between the centre of mass and the front axe (resp. rear axe).

The camber angles of front and rear wheel are given by:

$$
\left\{\begin{array}{l}
\phi_{f}=\phi+\delta \cdot \sin (\varepsilon) \\
\phi_{f}=\phi
\end{array}\right.
$$

Finally, we can express the lateral forces on the two wheels as follows:

$$
\left\{\begin{array}{l}
F_{y f}=C_{f 1}\left(\delta \cos (\varepsilon)-\frac{v_{y}+l_{f} \cdot \dot{\psi}-\eta \cdot \dot{\delta}}{v_{x}}\right)+C_{f 2}(\phi+\delta \sin (\varepsilon)) \\
F_{y r}=C_{r 1}\left(\frac{l_{r} \cdot \dot{\psi}-v_{y}}{v_{x}}\right)+C_{r 2} \cdot \phi
\end{array}\right.
$$


Under dynamical conditions, load can transfer to the front wheel when braking or to the rear wheel during acceleration.

When the vehicle is accelerating, normal forces on the two tires can be adapted ed as follows:

$$
\left\{\begin{array}{l}
F_{z f}=\frac{M_{f}+M r}{l_{1}+l_{2}}\left(l_{2} \cdot g \cdot \cos \left(\phi_{p}\right) \cdot \cos \left(\phi_{r}\right)-\bar{h} \cdot a_{x}\right) \\
F_{z r}=\frac{M_{f}+M r}{l_{1}+l_{2}}\left(l_{1} \cdot g \cdot \cos \left(\phi_{p}\right) \cdot \cos \left(\phi_{r}\right)+\bar{h} \cdot a_{x}\right)
\end{array}\right.
$$

where, $\bar{h}$ is the height of the center of mass, $l_{1}$ (resp. $l_{2}$ ) corresponding to the dimensions of vehicle configuration (horizontal dimensions of the overall center of gravity relative to the center of the front wheel, rear respectively). $F_{z f}$ (resp. $F_{z f}$ ) represents normal forces applied on the front tire (rear tire).

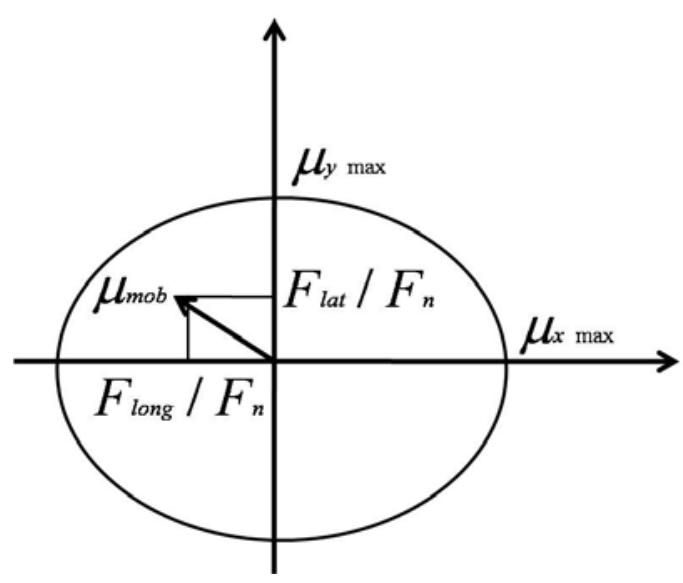

Fig. 3. Friction Ellipse [3]

\section{B. Tire Road Friction}

The tire is one of the main components of the vehicle. Indeed, it represents the interface of the aforementioned with the external environment which is the road. It transmits the guidance and braking/traction efforts [3]. The tire dynamic behavior is very complex and is linear only under certain restricted conditions of driving. One observes various phenomena like skidding and blocking.

The Coulomb friction model is used to obtain the mobilized friction $\mu_{m o b}$. Thus the transversal force $F_{t}$ and the normal force $F_{n}=F_{z f}+F_{z r}$ are such that:

$$
\mu_{m o b}=\frac{F_{t}}{F_{n}} \leq \mu_{\max }
$$

where $\mu_{\max }$ is the maximum available friction.

Since:

$$
F_{t}^{2}=F_{\text {lat }}^{2}+F_{\text {long }}^{2}
$$

we have:

$$
\left\{\begin{array}{l}
\mu_{\text {mob }}^{2}=\frac{F_{\text {lat }}^{2}+F_{\text {long }}^{2}}{F_{n}^{2}}=\mu_{\text {lat }}^{2}+\mu_{\text {long }}^{2} \\
\mu_{\text {lat }}=\frac{F_{\text {lat }}}{F_{n}} \\
\mu_{\text {long }}=\frac{F_{\text {long }}}{F_{n}}
\end{array}\right.
$$

Considering the following approximations of the yaw rate, the lateral and the longitudinal accelerations:

$$
\left\{\begin{array}{l}
\gamma_{\text {lat }}=\rho \cdot v_{x}^{2} \\
\gamma_{\text {long }}=\frac{d v_{x}}{d t}=v_{x} \frac{d v_{x}}{d s} \\
\dot{\psi}=\rho \cdot v_{x}
\end{array}\right.
$$

Under the assumption of the low values of the angles (superelevation and slope), and according to (5), (6) and (8) the expressions of the lateral and longitudinal frictions on each wheel are:

$$
\left\{\begin{array}{l}
\mu_{\text {lat } f}=\frac{C_{f 1}\left(v_{x} \delta \cos \varepsilon-v_{y}-\dot{\psi} l_{f}+\eta \dot{\delta}\right)-v_{x} C_{f 2}(\phi+\delta \sin \varepsilon)}{v_{x} \cdot A \cdot\left(B-h \cdot a_{x}\right) \cdot \mu_{\text {latmax }}} \\
\mu_{\text {latr }}=\frac{C_{r 1}\left(-v_{y}+\dot{\psi} l_{l}\right)}{v_{x} \cdot A \cdot\left(C+h \cdot a_{x}\right) \cdot \mu_{\text {latmax }}}
\end{array}\right.
$$

and:

$$
\left\{\begin{array}{l}
\mu_{\text {longf }}=\frac{K_{p 1} \cdot \lambda_{1}}{A \cdot\left(B-h \cdot a_{x}\right) \cdot \mu_{\text {longmax }}} \\
\mu_{\text {longr }}=\frac{K_{p 2} \cdot \lambda_{2}}{A \cdot\left(C+h \cdot a_{x}\right) \cdot \mu_{\text {longmax }}}
\end{array}\right.
$$

with :

$$
\left\{\begin{array}{l}
A=\frac{M_{f}+M_{r}}{l_{1}+l_{2}} \\
B=l_{2} \cdot g \cdot \cos \left(\phi_{p}\right) \cdot \cos \left(\phi_{r}\right) \\
C=l_{1} \cdot g \cdot \cos \left(\phi_{p}\right) \cdot \cos \left(\phi_{r}\right)
\end{array}\right.
$$

The highest safe speed is defined considering the maximum mobilized friction. As we consider a two-wheel vehicle model, we have to find the maximum of the two mobilized frictions, so:

$$
\mu_{\max }=\max \left(\sqrt{\mu_{\text {latf }}^{2}+\mu_{\text {longf }}^{2}}, \sqrt{\mu_{\text {latr }}^{2}+\mu_{\text {longr }}^{2}}\right)
$$

\section{Driver Behavior}

The driver is unceasingly obliged, according to the information taken from the environment, to define a speed and a position appropriate to the situation of control [3]. Here the ability of the driver in acceleration are taken into account. The driver cannot mobilize the same level of acceleration into longitudinal and lateral directions. For this reason we have to distinguish between the maximum friction in longitudinal and lateral modes: 


$$
\begin{gathered}
1=\max \left(\sqrt{\left(\frac{\mu_{\text {lat } f}}{\mu_{\text {latmax }}}\right)^{2}+\left(\frac{\mu_{\text {long } f}}{\mu_{\text {longmax }}}\right)^{2}},\right. \\
\left.\sqrt{\left(\frac{\mu_{\text {latr }}}{\mu_{\text {latmax }}}\right)^{2}+\left(\frac{\mu_{\text {longr }}}{\mu_{\text {longmax }}}\right)^{2}}\right)
\end{gathered}
$$

For comfort and safety reasons, and under good weather conditions, a driver generally does not mobilize all the available lateral and/or longitudinal friction; therefore we represent the driver behavior with through two dimensionless coefficients $\lambda_{\text {lat }}, \lambda_{\text {long }}$ and a maximum friction $\mu_{\max }$ [3].

$$
\mu_{\text {latmax }}=\lambda_{\text {lat }} \cdot \mu_{\max } \quad \text { and } \quad \mu_{\text {longmax }}=\lambda_{\text {long }} \cdot \mu_{\text {max }}
$$

It is well known that driver behavior adapts according to maximum available friction. We considered that the driver behavior varies inversely proportional to maximum friction $\mu_{\text {max }}$.

$$
\left\{\begin{array}{l}
\lambda_{\text {long }}=\Delta \lambda_{\text {long }} \cdot \mu_{\text {max }}-\lambda_{\text {slip-long }} \\
\lambda_{\text {lat }}=\Delta \lambda_{\text {lat }} \cdot \mu_{\text {max }}-\lambda_{\text {slip-lat }}
\end{array}\right.
$$

with:

$$
\left\{\begin{array}{l}
\Delta \lambda_{\text {long }}=\lambda_{\text {dry-long }}-\lambda_{\text {slip-long }} \\
\Delta \lambda_{\text {lat }}=\lambda_{\text {dry-lat }}-\lambda_{\text {slip-lat }}
\end{array}\right.
$$

where, $\lambda_{d r y}$ (resp. $\lambda_{\text {slip }}$ ) represents the quantity of maximum friction that the driver can mobilize on a dry road (resp. slipping road).

\section{Generation of Speed Profile}

By developing the equations (16) and (17), and after the calculation of the safe speeds at each of the wheels:

$$
\left\{\begin{array}{l}
v_{1}=\frac{-\Delta_{1}+\Delta_{3} \sqrt{1-\Delta_{4}^{2}}}{\Delta_{2}} \\
v_{2}=\frac{-\Delta_{1}-\Delta_{3} \sqrt{1-\Delta_{4}^{2}}}{\Delta_{2}}
\end{array}\right.
$$

with:

$$
\left\{\begin{aligned}
\Delta_{1} & =C_{r 2} \cdot \phi \\
\Delta_{2} & =C_{r 1}\left(b \cdot \dot{\psi}-v_{y}\right) \\
\Delta_{3} & =A\left(C-h \cdot a_{x}\right) \cdot \mu_{\text {latmax }} \\
\Delta_{4} & =A\left(C-h \cdot a_{x}\right) \cdot \mu_{\text {longmax }}
\end{aligned}\right.
$$

Then, the final safe speed $\mathrm{V}$ in a curve is obtained from: $V=\min \left(V_{1}, V_{2}\right)$.

This model of safe speed in curve is function of intrinsic parameters (mass, inertia), extrinsic parameters (road geometry, available friction) and driver behavior (driver ability in deceleration). It takes into account the load transfer and the vehicle side slip, and longitudinal dynamics by considering the longitudinal slip on each wheel.

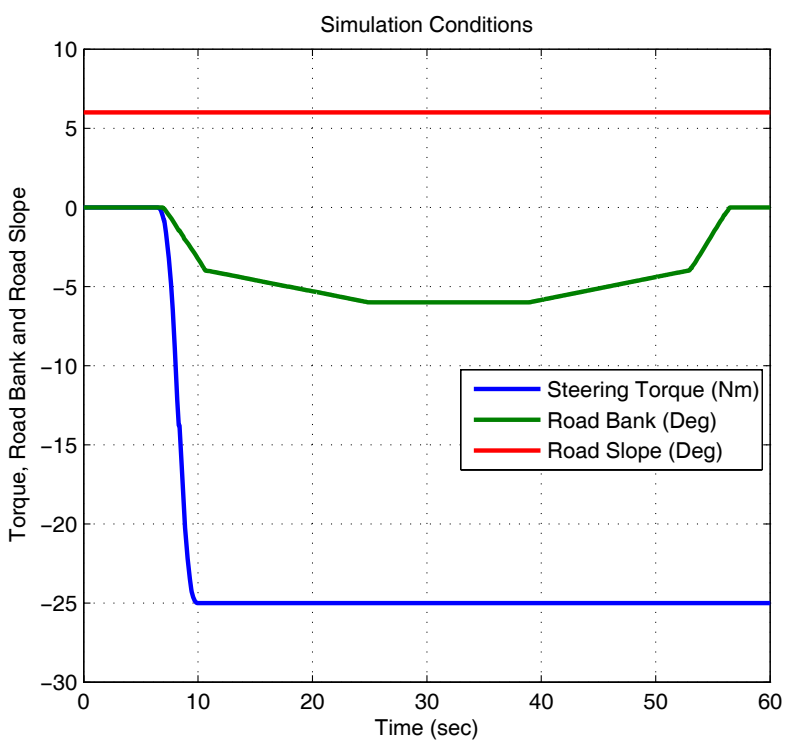

Fig. 4. Steering torque, road bank and slope

\section{Simulation Results}

In order to illustrate the effectiveness of the proposed procedure, different simulation tests are conducted. Two sets of simulation will be performed with and without road bank angle.

Simulation condictions : steering torque, road banking and slope angles, are represented in figure 4.

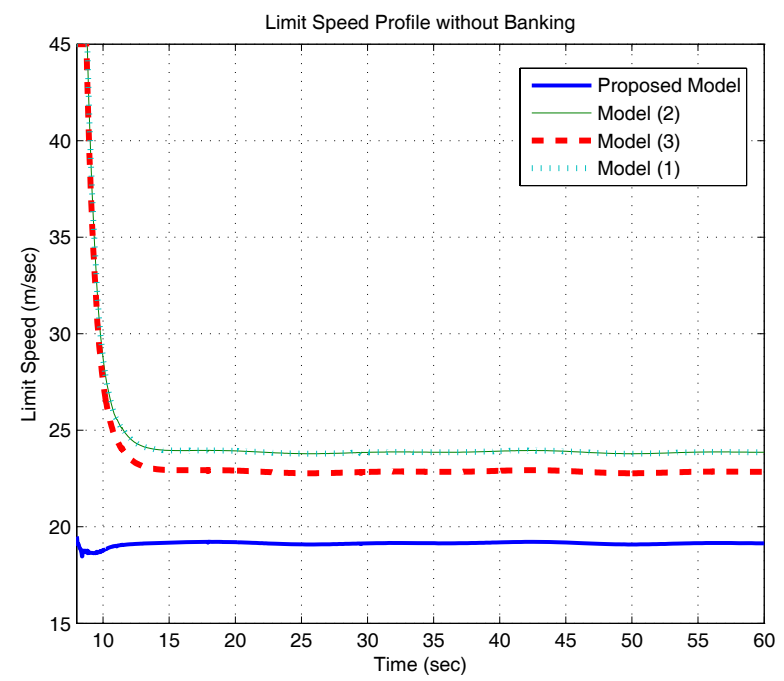

Fig. 5. Limit speed profile without banking

Figure 5 shows limit speed profile by respect to 4 models represented respectively by equations $1,2,3,25$. In the case where, the motorcycle operates on a flat road without banking, the previous models differ, with more restrictions to the proposed model which recommends for maximum 
speed about $(19 \mathrm{~m} / \mathrm{sec})$, to negotiate the turn safely.

With road banking angle, the simulation results show (Figure 5) a clear difference on the limit speed profile for the previous models. We can notice the increase of different limits speed computed (Figure 6) on the portions of the road with banking. This due to the road bank angle which absorb a part of the lateral acceleration.

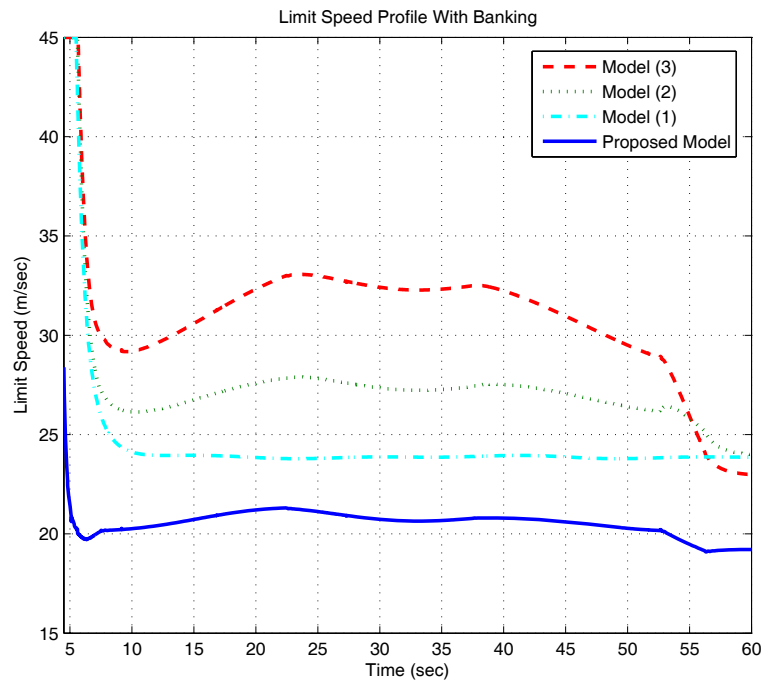

Fig. 6. Limit speed profile with banking

\section{CONCLUSIONS AND FUTURE WORKS}

In this paper, a new method for the calculation of the highest safe speed for driving the upcoming curve was proposed. This safe speed model takes into account the infrastructure characteristics using an accurate description of the road geometry (radius of curvature, slope and superelevation), and the estimated maximal available friction, the vehicle dynamics and driver behavior. Parameter effects analysis has been performed. Simulation results show clearly the effectiveness of this approach.

In the future, an instrumented scooter will be used to validate the generated speed profiles, and to ajust optimal thresholds for warning generation. Moreover, the involvement of the vehicle dynamics can be wider, by using observers to estimate the longitudinal slip and the vehicle side slip angles. These aspects will be incorporated in prototype vehicle and will be tested.

\section{REFERENCES}

[1] H. Slimi, H. Arioui, L. Nouveliere and S. Mammar, "Advanced Motorcycle-Infrastructure-Driver Roll Angle Profile for Loss Control Prevention", 12th International IEEE Conference on Intelligent Transportation Systems., St. Louis, Missouri, U.S.A., October 3-7, 2009

[2] B. Amans, M. Moutreuil, "RIDER : Recherche sur les accidents impliquant un deux roues motorisé", Reaserch Report of RIDER Project, Mars 2005.
[3] Glaser, S., 2004, “ Modélisation et analyse d'un véhicule en trajectoire limites Application au développement de systèmes d'aide á la conduite". PhD thesis, University of Evry Val d'Essonne (France).

[4] C. Koenen, "The dynamic behaviour of motorcycles when running straight ahead and when cornering," Ph.D. dissertation, Delft University of Technology, 1983.

[5] D. J. N. Limebeer and R. S. Sharp, "Bicycles, motorcycles and models," IEEE Control Systems magazine, vol. 26, no. 5, pp. 34-61, 2006.

[6] R. S. Sharp and D. J. N. Limebeer, "A motorcycle model for stability and control analysis,"Multibody System Dynamics, vol. 6, no. 2, pp. 123-142, 2001.

[7] V. Cossalter and R. Lot, "A motorcycle multi-body model for real time simulations based on the natural coordinates approach,"Vehicle System Dynamics", vol. 37, no. 6, pp. 423-447, 2002.

[8] H. Slimi, H. Arioui, L. Nouveliere and S. Mammar, "Preventive Safety: Warning System for Control Loss of Two-Wheeled Vehicles", Informatics, Integrative Biology and Complex Systems, Zarzis, Tunisia on 23-25 March 2009.

[9] S Hima, L Nehaoua, N Séguy and H Arioui, "Motorcycle Dynamic Model Synthesis for Two Wheeled Driving Simulator", IEEE Intelligent Transportation Systems Conference, pp. 812-817, 2007.

[10] Hans B. Pacejka, Tyre and Vehicle Dynamics, p 511- 562 , Delft University of Technology, 2002.

[11] W.D. Versteden, Improving a tyre model for motorcycle simulations, Maters Thesis, Technical University of Eindhoven, June 2005.

[12] S. Kidane, L. Alexander, R. Rajamani, P. Starr and M. Donath, Road Bank Angle Considerations in Modeling and Tilt Stability Controller Design for Narrow Commuter Vehicles, Department of Mechanical Engineering, University of Minnesota, Minneapolis, USA, June 1416, 2006.

[13] D. Karnopp, and R. Hibbard. Optimum Roll Angle behavior for Tilting Ground Vehicle, DSC, Vol. 44, 1992. $\phi, \psi, \delta$

$F_{y f}, F_{x f}$

$F_{y r}, F_{x r}$

$F_{z f}, F_{z r}$

$x, y$

$M, M_{f}, M_{r}$

$\phi_{p}, \phi_{r}$

$\rho$

$a_{i}, b_{i}, c_{i}$

$\alpha_{f}, \alpha_{r}$

$\theta_{f}, \theta_{r}$

$\tau$

$\kappa$

$\eta$

$\varepsilon$

$g$

$j, k, h, L_{f}, L_{r}, e$

$\lambda_{1}, \lambda_{1}$

\section{APPENDIX}

Roll, yaw and direction angles

Lateral and longitudinal front forces

Lateral and longitudinal rear forces

Front and rear vertical forces

Longitudinal and lateral displacement

Total, front and rear masses

Slope and road bank angles

Parameters

Front and rear sideslip angles

Front and rear camber angles

Rider applied steering torque

Steering damper coefficient

Trail

Caster angle

Acceleration due to gravity

dimensional parameters (Figure 2)

Front and rear longitudinal slip
Curvature 